\title{
What factors are associated with excess body weight in Australian secondary school students?
}

\section{Belinda C Morley $\mathrm{PhD}, \mathrm{MPH}$, Obesity Prevention Research and Evaluation Manager \\ Maree L Scully BA(Hons) Research Officer}

Philippa H Niven BAppSc(Psych)(Hons) Research Assistant

Anthony D Okely EdD, BEd(Hons)
Director

Louise A Bau BSc(Med), PhD, FRACP Professor ${ }^{3}$

lain S Pratt GradDip(Diet), APD, AEP, Nutrition and Physical Activity Manager

Melanie A Wakefield BA, MA, PhD Director

on behalf of the NaSSDA study team*

1 Centre for Behavloural Research in Cance Cancer Council Victoria, Melbourne, VIC.

2 Interdisciplinan Educational Research Institute,

University of Wollongong Wollongong, NSW.

3 Discipline of Paediatrics and Child Health University of Sydney, Sydney, NSW.

4 Cancer Council Western Australia, Perth, WA.

melanie.wakefield@ cancervic.org.au

* The NaSSDA Study Team comprises Cancer Counci Victoria: Belinda Morley, Maree Scully, Melanie Wakefield Technical Advisory Group: Louise Baur (Chair) Anthony Loulse Baur (Chair), Anthon Jo Salmon, Victoria Flood,
J lain Pratt, Jane Bowen, David Crawford Anthony Worsley.

MJA 2012; 196: 189-192 doi: 10.5694/mjall.11184

Editorial p 152 Perspective p 165 Letter $\mathbf{p} 171$ Clinical focus p 174 Research p 184

\section{T} he prevalence of overweight and obesity among Australian children and adolescents doubled between 1985 and 1997,, with similar increases observed in other large developed countries. ${ }^{2}$ Young people who are overweight or obese are at increased risk of experiencing chronic diseases such as type 2 diabetes, cardiovascular disease, stroke and some cancers later in life. ${ }^{3}$

While many studies have focused on exploring the link between one specific behaviour (eg, excessive time spent watching television, short sleep duration) and overweight or obesity in this population, only a few have assessed the relative importance of a range of factors that are postulated to be associated with excess body weight., ${ }^{4,5}$ Given the finite resources available to tackle obesity, a clearer understanding of the correlates of overweight in adolescence is needed to allow preventive efforts to be directed towards areas where they are likely to have the most benefit.

The aim of our study was to describe the current prevalence of overweight and obesity among Australian adolescents and to examine associations between weight status and selected demographic and health-behaviour characteristics in order to identify potential targets for intervention.

\section{Methods}

We used data from the National Secondary Students' Diet and Activity (NaSSDA) survey conducted in Australia during May 2009 to April 2010. The methods employed in the NaSSDA survey have been described in detail elsewhere. ${ }^{6}$ In brief, the sampling procedure was a two-stage probability design, with schools randomly selected at the first stage of sampling and classes selected within schools at the second stage. Students

\begin{abstract}
Objectives: To examine the prevalence of overweight and obesity in Australian secondary school students and identify factors associated with excess adiposity.
\end{abstract}

Design, setting and participants: Cross-sectional survey of students aged 12-17 years (in school years 8-11) who completed the National Secondary Students' Diet and Activity survey in 2009-10, which included a web-based self-report questionnaire and height and weight measurements.

Main outcome measures: Overweight and obesity based on international standard body mass index (BMI) cut-offs for children and adolescents.

Results: Data were analysed for 12188 students. Just under one in four students were either overweight (18\%) or obese (5\%). After adjusting for demographic and health-behaviour characteristics, males were more likely than females to be overweight or obese (OR, 1.23; 95\% Cl, 1.07-1.40; $P=0.004)$, as were both low (OR, 1.67; 95\% Cl, 1.40-1.99; $P<0.001$ ) and medium (OR, 1.33; 95\% Cl, 1.14-1.55; $P<0.001$ ) socioeconomic position (SEP) students compared with high SEP students. Students engaging in low levels of physical activity (OR, $1.21 ; 95 \% \mathrm{Cl}$, 1.08-1.36; $P=0.001)$, more time in small-screen recreation (OR, $1.18 ; 95 \% \mathrm{Cl}$, $1.05-1.32 ; P=0.005$ ), and short sleep duration (OR, 1.22; $95 \% \mathrm{Cl}, 1.05-1.41 ; P=$ 0.008 ) also had higher odds of being overweight or obese.

Conclusions: There is a need for interventions to reduce overweight and obesity during adolescence. Preventive measures should include a focus on facilitating physical activity and reducing sedentary behaviour, as well as promoting adequate sleep, particularly among young people from lower SEP neighbourhoods who appear to be most susceptible.

completed a web-based questionnaire assessing their diet, physical activity and sedentary behaviour in their regular class groups, and had their height and weight measured by trained researchers in a confidential setting. The survey was approved by the Human Research Ethics Committee of Cancer Council Victoria, relevant education authorities in each state and territory, and the principals of selected schools. Informed written parent or carer and student consent was required for participation in each component of the study.

\section{Measures}

\section{Questionnaire}

Consumption of key healthy or core foods was assessed by asking students to indicate how many serves of vegetables and fruit they usually ate each day. These short dietary questions were used in the 1995 National Nutrition Survey and have reasonable validity when compared with 24 -hour recall of vegetable and fruit intake for adults. $^{7}$ Students were identified as being low-level consumers of vegetables or fruit if they reported eating one serve or less per day of each.

Intake of key unhealthy or noncore foods was assessed by asking students to indicate how frequently they consumed sugary drinks (eg, soft drink, cordials, sports drinks) and how often they had meals or snacks such as burgers, pizza, chicken or chips from fast-food or takeaway food places. Students were identified as being high consumers of fast food if they reported eating these foods at least 1-2 times a week, and of sugary drinks if they reported drinking at least one cup (250 ml) per day.

Physical activity was assessed using the 60-minute moderate-to-vigorous physical activity (MVPA) screening measure, which is reliable, valid and correlates well with objective measures of physical activity. ${ }^{8}$ Students were identified as engaging in low levels of physical activity if they did not meet the recommended $60 \mathrm{~min}$ utes of MVPA per day on at least 4 days in the past week. 
Time spent in small-screen recreation was assessed using a subscale of the Adolescent Sedentary Activity Questionnaire, which has high reliability. ${ }^{9}$ Students indicated how long they usually spent watching television, videos and DVDs, playing video games, and using the computer for fun on school days and the weekend. Responses were combined to identify students who reported spending, on average, 4 or more hours per day (ie, a high amount of time) in small-screen recreation.

Students' sleeping patterns were assessed by asking them to record the time they went to bed and turned the light out the previous night and the time they woke up that morning, or, if either time was not "usual", the time they usually went to bed on a school night or woke up on a school day. Sleep duration was calculated as the difference between usual wakeup time and bedtime (in minutes), and dichotomised to identify students who slept for 8 hours or less on school nights. This cut-point was chosen to align with the lowest category used in an earlier Australian study. ${ }^{10}$

\section{Anthropometry}

Measurements of students' height and weight were taken in accordance with standardised protocols. ${ }^{11}$ Height was measured using portable stadiometers (Model IP0955; Invicta Plastics, Leicester, UK). Weight was measured using digital scales (Model UM051; Tanita, Tokyo, Japan). Height and weight measurements were used to calculate body mass index (BMI $\left.\left[\mathrm{kg} / \mathrm{m}^{2}\right]\right)$, and students were classified as overweight or obese according to cut-offs developed for children and adolescents by the International Obesity Task Force. ${ }^{12}$ Cole and colleagues' BMI cut-offs to define grade 1 thinness in children and adolescents were used to classify students as underweight. ${ }^{13}$

\section{Demographics}

Students' sex, school year and home postcode were recorded. A measure of low, medium or high socioeconomic position (SEP) was determined ${ }^{6}$ according to the Socio-Economic Index for Areas Index of Relative Socio-economic Disadvantage, based

1 Body mass index (BMI) distribution among Australian secondary students,* by $\operatorname{sex}(n=12188)$

\begin{tabular}{lccc} 
& Males, \% (95\% Cl) & Females, \% (95\% Cl) & All, \% (95\% Cl) \\
\hline Underweight $^{\dagger}$ & $4.3 \%(3.6 \%-5.1 \%)$ & $5.4 \%(4.7 \%-6.2 \%)$ & $4.8 \%(4.2 \%-5.5 \%)$ \\
\hline Healthy weight & $71.2 \%(69.4 \%-72.9 \%)$ & $72.6 \%(70.6 \%-74.5 \%)$ & $71.8 \%(70.4 \%-73.2 \%)$ \\
Overweight $^{\ddagger}$ & $19.2 \%(17.5 \%-21.1 \%)$ & $17.4 \%(16.1 \%-18.8 \%)$ & $18.4 \%(17.3 \%-19.5 \%)$ \\
Obese $^{\ddagger}$ & $5.4 \%(4.5 \%-6.3 \%)$ & $4.6 \%(3.7 \%-5.7 \%)$ & $5.0 \%(4.3 \%-5.8 \%)$ \\
\hline
\end{tabular}

Percentages are rounded, so may not sum to $100 \%$. * Sample comprised students participating in the National Secondary Students' Diet and Activity survey conducted in Australia in 2009-10. † Based on Cole and colleagues' BMI index cut-offs to define grade 1 thinness in children and adolescents. ${ }^{13}$

$\ddagger$ Based on the International Obesity Task Force BMI index cut-offs to define overweight and obesity in children and adolescents. ${ }^{12}$

on each student's home postcode. Postcode of residence was also used to classify the geographical location of students as either metropolitan or rural/regional.

\section{Data analyses}

We analysed the data using Stata SE, version 11.1 (StataCorp, College Station, Tex, USA), with weighting for state, education sector, school year and sex to ensure the sample reflected the population distribution. ${ }^{14}$ We used multiple logistic regression analysis to explore the extent to which students' sex, school year, SEP, geographical location, eating behaviour, physical activity levels, small-screen recreation habits and sleeping patterns were independently related to their being overweight or obese. All analyses adjusted for the clustering of students within each school. Because of the large sample size, we applied a more conservative level of statistical significance $(P<0.01)$.

\section{Results}

A total of 238 secondary schools participated (a response rate of 39\%) Data were collected from 14066 students (a student response rate of $54 \%$ ). Students were excluded from analyses if they had not participated in the physical measurements (1515) or had not completed at least $33 \%$ of the survey questions (223). A further 140 students were also excluded because they were not aged 12 to 17 years on the day of the survey or no SEP index value was available for their postcode. We analysed data for the remaining 12188 students.

Overall, 53\% were male and slightly higher proportions were in Years $8(31 \%)$ and $9(27 \%)$ compared with Years 10 (22\%) and $11(20 \%)$.
Thirty-two per cent resided in low SEP neighbourhoods and $26 \%$ were from high SEP neighbourhoods. Nearly two-thirds of students (64\%) lived in metropolitan areas.

The BMI distributions for males, females and overall are shown in Box 1.

Adjusted prevalence and odds of overweight or obesity associated with each of the selected demographic and health-behaviour characteristics are shown in Box 2. Preliminary logistic regression analyses were conducted with all covariates as well as the interaction terms: vegetable and fruit consumption; intake of sugary drinks and fast food; and levels of physical activity and small-screen recreation. None of the interactions were significant, and they were removed from the final model. To assess collinearity, separate logistic regression analyses were conducted to examine bivariate associations between being overweight or obese and each health-behaviour characteristic. As these results were consistent with the significant associations that were found in the final model, we concluded that collinearity was not a cause for concern. After adjustment for all covariates in the model, sex, SEP, physical activity, small-screen recreation and sleep duration were found to be independently associated with overweight or obesity. Specifically, males were more likely than females to be classified as overweight or obese (odds ratio [OR], 1.23; 95\% CI, 1.07-1.40; $P=0.004$ ). There was also a strong gradient related to SEP, with students from low-SEP neighbourhoods (OR, 1.67; 95\% CI, 1.40-1.99; $P<0.001)$ and medium-SEP neighbourhoods (OR, $1.33 ; 95 \% \mathrm{CI}, 1.14-1.55 ; P<0.001)$ being more likely to be overweight or obese compared with students residing in high-SEP neighbourhoods. In 
addition, the odds of being overweight or obese were higher among students who were not sufficiently active on at least 4 days in the past week (OR, $1.21 ; 95 \%$ CI, 1.08-1.36; $P=0.001)$ and those who engaged in small-screen recreation for at least 4 hours per day (OR, 1.18; 95\% CI, 1.05-1.32; $P=$ $0.005)$. Sleeping for 8 hours or less on a usual school night was also associated with increased odds of being overweight or obese (OR, 1.22; 95\% CI, 1.05-1.41; $P=0.008)$.

\section{Discussion}

The results of our study indicate that nearly one in four Australian secondary school students (23\%) are categorised as overweight or obese, based on measured height and weight. This is comparable to recent national data using different age ranges and methods that consistently show that around a quarter of Australian children and adolescents are overweight or obese. ${ }^{15,16}$ Importantly, our study builds on these data by replicating the finding in a considerably larger sample of young Australians. While these previous national surveys reported no significant differences by sex, 15,16 our multiple logistic regression analyses indicated a higher prevalence of overweight and obesity among male students. Although there is debate about whether childhood rates of overweight and obesity in Australia may be stabilising, ${ }^{17}$ it is unequivocal that the current prevalence estimate for adolescents is still of concern.

A clear gradient related to SEP was evident, as the prevalence of overweight and obesity increased with declining SEP. Indeed, SEP emerged as the strongest factor associated with overweight or obesity among Australian secondary school students after taking into account other demographic and health-behaviour characteristics. Similar inverse associations between SEP and adiposity in young people have been found in previous studies conducted in Australia and other developed countries. ${ }^{18}$ While our study only included one area-based marker of SEP, Canadian research has indicated that both individual and arealevel measures of SEP are independently related to obesity in adoles- cents. ${ }^{19}$ Thus, further exploratory work that incorporates a number of SEP markers (eg, parental education, family income), as well as environmental characteristics such as density of parks and neighbourhood walkability, is needed to help explain the observed relationship between low SEP and overweight. Nonetheless, future obesity-prevention strategies should aim to redress this social disparity.

Low physical activity levels and greater time spent in small-screen recreation were both independently associated with overweight or obesity among Australian adolescents. This is consistent with findings from a large school-based cross-sectional survey that was conducted in 34 countries among students aged 10-16 years. In most countries, after controlling for known dietary predictors, lower physical activity participation and higher television viewing time were associated with increased odds of being overweight. ${ }^{5}$ Australian guidelines recommend that adolescents engage in at least 60 minutes of moderate to vigorous physical activity every day and spend no more than 2 hours a day using electronic media for entertainment. ${ }^{20}$ Creating supportive environments for physical activity may help to promote increased physical activity among young people and could possibly displace time spent in smallscreen recreation.

In this study, dietary factors such as low vegetable and fruit intake and high consumption of sugary drinks and fast food were unrelated to overweight status among Australian adolescents. This result is again similar to the multinational school survey, although their analyses did not include intake of fast food in the regression models. ${ }^{5}$ Other studies have produced mixed results regarding the link between fast-food consumption and excess weight. ${ }^{21}$ The relationship between diet and body weight is extremely complex and requires a more thorough assessment of dietary factors, such as eating patterns, portion sizes, and individual nutrient intake, than was possible in our study.

There was strong evidence of a link between weight status and short sleep duration. Previous Australian research has found an inverse relationship
2 Adjusted odds ratios* and prevalences of overweight or obesity among Australian secondary students, ${ }^{\dagger}$ by selected demographic and behavioural characteristics $(n=11984)$

\begin{tabular}{|c|c|c|c|c|}
\hline Covariate & No. & $\begin{array}{l}\text { Adjusted odds ratio } \\
\qquad(95 \% \mathrm{Cl})\end{array}$ & $P^{\ddagger}$ & $\begin{array}{c}\text { Adjusted } \\
\text { prevalence }\end{array}$ \\
\hline \multicolumn{5}{|l|}{ Sex } \\
\hline Male & 6338 & $1.23(1.07-1.40)$ & 0.004 & $25.1 \%$ \\
\hline Female & 5646 & ref & & $21.5 \%$ \\
\hline \multicolumn{5}{|l|}{ School year } \\
\hline 8 & 3703 & ref & & $23.3 \%$ \\
\hline 9 & 3281 & $1.00(0.88-1.14)$ & 0.964 & $23.3 \%$ \\
\hline 10 & 2595 & $0.96(0.83-1.11)$ & 0.575 & $22.6 \%$ \\
\hline 11 & 2405 & $1.06(0.90-1.25)$ & 0.482 & $24.3 \%$ \\
\hline \multicolumn{5}{|c|}{ Socioeconomic position } \\
\hline Low & 3840 & $1.67(1.40-1.99)$ & $<0.001$ & $27.1 \%$ \\
\hline Medium & 4986 & $1.33(1.14-1.55)$ & $<0.001$ & $22.9 \%$ \\
\hline High & 3158 & ref & & $18.3 \%$ \\
\hline \multicolumn{5}{|c|}{ Geographical location } \\
\hline Metropolitan & 7711 & ref & & $23.8 \%$ \\
\hline Rural & 4273 & $0.94(0.79-1.12)$ & 0.497 & $22.8 \%$ \\
\hline \multicolumn{5}{|c|}{ Vegetables (usual daily serves) } \\
\hline$\leqslant 1$ & 2903 & $0.97(0.82-1.15)$ & 0.734 & $23.0 \%$ \\
\hline$\geqslant 2$ & 9081 & ref & & $23.5 \%$ \\
\hline \multicolumn{5}{|c|}{ Fruit (usual daily serves) } \\
\hline$\leqslant 1$ & 3357 & $0.86(0.76-0.98)$ & 0.029 & $21.6 \%$ \\
\hline$\geqslant 2$ & 8627 & ref & & $24.1 \%$ \\
\hline \multicolumn{5}{|c|}{ Sugary drinks (usual frequency) } \\
\hline$\leqslant 4-6$ cups/wk & 10378 & ref & & $23.3 \%$ \\
\hline$\geqslant 1-2$ cups/day & 1606 & $1.04(0.88-1.22)$ & 0.636 & $24.0 \%$ \\
\hline \multicolumn{5}{|c|}{ Fast food (usual frequency) } \\
\hline < Once a week & 6838 & ref & & $24.5 \%$ \\
\hline$\geqslant 1-2$ times/wk & 5146 & $0.86(0.74-1.00)$ & 0.057 & $21.9 \%$ \\
\hline \multicolumn{5}{|c|}{ Physical activity (no. of days sufficiently active over past week) } \\
\hline $0-3$ & 5497 & $1.21(1.08-1.36)$ & 0.001 & $25.2 \%$ \\
\hline $4-7$ & 6487 & ref & & $21.8 \%$ \\
\hline \multicolumn{5}{|c|}{ Small-screen recreation (no. of hours/day) } \\
\hline$<4$ & 6106 & ref & & $21.9 \%$ \\
\hline$\geqslant 4$ & 5878 & $1.18(1.05-1.32)$ & 0.005 & $24.8 \%$ \\
\hline \multicolumn{5}{|c|}{ Sleep duration (no. of hours/school night) } \\
\hline$\leqslant 8$ & 3055 & $1.22(1.05-1.41)$ & 0.008 & $26.0 \%$ \\
\hline$>8$ & 8929 & ref & & $22.4 \%$ \\
\hline
\end{tabular}

*Adjusted for all covariates, and clustering by school. † Sample comprised students participating in the National Secondary Students' Diet and Activity survey conducted in Australia in 2009-10. $¥ P<0.01$ significant.

between sleep duration and overweight among males aged $7-15$ years, but not among females. ${ }^{10}$ However, that study did not adjust for important covariates such as physical activity or time spent in screen-based sedentary recreation, diet and SEP. More recently, an association between short sleep duration and increased risk of obesity has been reported among South Australian children aged 5-15 years, after controlling for these covariates. ${ }^{22}$ While the current international literature is generally in support of a link between sleep duration 
and overweight status in children and adolescents, the mechanisms driving this association have not been clearly established but are likely to be multifactorial. ${ }^{23}$ Despite this, encouraging young people to get regular, sufficient sleep could represent a cost-effective intervention aimed at obesity prevention.

There are some study limitations that should be noted. Firstly, as this was a cross-sectional survey and weight status is a lag measure, no causal relationships can be inferred. Secondly, given the use of an active consent procedure, the rate of student participation was low, although the response rate was comparable to similar state-based school surveys recently undertaken in Australia. ${ }^{24,25}$ Further, nearly $11 \%$ of students who completed the online questionnaire did not consent to having their physical measurements taken. It is possible that those who are overweight or obese may have been more likely to opt out of being measured or to opt out of the study as a whole, thus leading to underestimates of prevalence. Important strengths of the study are that it was based on a large, representative sample of Australian secondary students, and that BMI prevalence data were derived from actual height and weight measurements of students rather than selfreport or parent-proxy estimates.

In conclusion, the proportion of Australian adolescents who are overweight or obese is of concern and highlights the need for intervention at this critical developmental phase. These findings suggest that preventive measures should include a particular focus on facilitating physical activity and reducing sedentary behaviour, as well as promoting adequate sleep. It is also important for future efforts to target young people from lower SEP neighbourhoods, who appear to be most susceptible to overweight.

Acknowledgements: The National Secondary Students' Diet and Activity Survey was jointly funded by state Cancer Councils through Cancer Council Australia, and by the National Heart Foundation of Australia. Melanie Wakefield is supported by an National Health and Medical Research Council Principal Research Fellowship. I-view was responsible for fieldwork coordination. We thank the school principals, teachers and students who participated in this study.

Competing interests: No relevant disclosures. There was no involvement of the study sponsors in study design, data collection, analysis and interpretation of data, the writing of the manuscript, or decision to submit for publication.

Received 19 Sep 2011, accepted 24 Jan 2012.

1 Booth ML, Chey T, Wake M, et al. Change in the prevalence of overweight and obesity among young Australians, 1969-1997. Am J Clin Nutr 2003: 77: 29-36.

2 Wang Y, Lobstein T. Worldwide trends in childhood overweight and obesity. Int J Pediatr Obes 2006; 1: 11-25.

3 World Health Organization. Diet, nutrition and the prevention of chronic diseases: report of a joint WHO/FAO expert consultation. WHO Technical Report Series, 916. Geneva: WHO, 2003.

4 Patrick K, Norman GJ, Calfas KJ, et al. Diet, physical activity, and sedentary behaviors as risk factors for overweight in adolescence. Arch Pediatr Adolesc Med 2004; 158: 385-390.

5 Janssen I, Katzmarzyk PT, Boyce WF, et al. Comparison of overweight and obesity prevalence in school-aged youth from 34 countries and their relationships with physical activity and dietary patterns. Obes $\operatorname{Rev} 2005 ; 6$ : 123-132.

6 Scully M, Wakefield M, Niven P, et al. Association between food marketing exposure and adolescents' food choices and eating behaviors. Appetite 2011; 58: 1-5.

7 Rutishauser IHE, Webb K, Abraham B, Allsopp R. Evaluation of short dietary questions from the 1995 National Nutrition Survey. Canberra: Australian Food and Nutrition Monitoring Unit, 2001.

8 Prochaska JJ, Sallis JF, Long B. A physical activity screening measure for use with adolescents in primary care. Arch Pediatr Adolesc Med 2001; 155 554-559.

9 Hardy LL, Booth ML, Okely AD. The reliability of the Adolescent Sedentary Activity Questionnaire (ASAQ). Prev Med 2007; 45: 71-74.

10 Eisenmann JC, Ekkekakis P, Holmes M. Sleep duration and overweight among Australian children and adolescents. Acta Paediatr 2006; 95: 956-963.

11 Davies PSW, Roodveldt R, Marks G. Standard methods for the collection and collation of anthropometric data in children. National Food and Nutrition Monitoring and Surveillance Project. Canberra: Commonwealth of Australia, 2001.

12 Cole TJ, Bellizzi MC, Flegal KM, Dietz WH. Establishing a standard definition for child overweight and obesity worldwide: international survey. BMJ 2000; 320: 1-6.

13 Cole TJ, Flegal KM, Nicholls D, Jackson AA. Body mass index cut offs to determine thinness in children and adolescents: international survey. BMJ 2007; 335: 194-201.

14 Australian Bureau of Statistics. Schools, Australia, 2009. Canberra: ABS, 2010. (ABS Cat. No. 4221.0.)

15 Australian Government Department of Health and Ageing. Australian Food and Grocery Council, Australian Government Department of Agriculture, Fisheries and Forestry. 2007 Australian National Children's Nutrition and Physical Activity Survey: main findings. Canberra: Commonwealth of Australia, 2008.

16 Australian Bureau of Statistics. National Health Survey: summary of results, 2007-2008. Canberra: ABS, 2009. (ABS Cat. No. 4364.0.)

17 Gill TP, Baur LA, Bauman AE, et al. Childhood obesity in Australia remains a widespread health concern that warrants population-wide prevention programs. Med J Aust 2009; 190: 146-148.

18 Shrewsbury V, Wardle J. Socioeconomic status and adiposity in childhood: a systematic review of cross-sectional studies 1990-2005. Obesity 2008; 16: 275-284.

19 Janssen I, Boyce WF, Simpson K, Pickett W. Influence of individual- and area-level measures of socioeconomic status on obesity, unhealthy eating, and physical inactivity in Canadian adolescents. Am J Clin Nutr 2006; 83: 139-145.

20 Australian Government Department of Health and Ageing. Australia's physical activity recommendations for $12-18$ year olds. Canberra: DoHA, 2004.

21 Rosenheck R. Fast food consumption and increased caloric intake: a systematic review of a trajectory towards weight gain and obesity risk. Obes Rev 2008; 9: 535-547.

22 Shi Z, Taylor AW, Gill TK, et al. Short sleep duration and obesity among Australian children. BMC Public Health 2010; 10: 609.

23 Hart CN, Jelalian E. Shortened sleep duration is associated with pediatric overweight. Behav Sleep Med 2008; 6: 251-267.

24 Martin K, Rosenberg M, Miller M, et al. Move and Munch. Trends in physical activity, nutrition and body size in Western Australian children and adolescents: the Child and Adolescent Physical Activity and Nutrition Survey (CAPANS) 2008. Final report. Perth: Western Australian Government, 2010.

25 Hardy LL, King L, Espinel P, et al. Methods of the NSW Schools Physical Activity and Nutrition Survey 2010 (SPANS 2010). J Sci Med Sport 2011; 14: 390-396. 